\title{
Treatment patterns and clinical outcomes of palbociclib-based therapy received in US community oncology practices
}

\author{
Junji Lin*,1 (D), Lynn McRoy², Maxine D Fisher ${ }^{1}, \mathrm{Nan} \mathrm{Hu}^{1}$, Cralen Davis ${ }^{1}$, Debanjali Mitra ${ }^{2}$ \& \\ Mark S Walker ${ }^{1}$ \\ ${ }^{1}$ Vector Oncology, an affiliate of ConcertAl, 6555 Quince, Suite 400, Memphis, TN 38119, USA \\ 2Pfizer Inc., 235 East 42nd Street, NY 10017, USA \\ *Author for correspondence: junjilin@gmail.com
}

Background: Limited studies have evaluated palbociclib-based therapy use in patients with advanced/metastatic breast cancer in the real world. This retrospective study used medical records from US community oncology practices to address the gap. Materials \& methods: Eligible patients receiving palbociclib-based therapy per label indication from 3 February 2015 to 31 December 2017 were included. Descriptive analyses were conducted for patient characteristics, treatment patterns and clinical outcomes. Results: The study included 233 patients who received palbociclib + aromatase inhibitor $(\mathrm{P}+\mathrm{Al})$ and 48 who received palbociclib + fulvestrant $(\mathrm{P}+\mathrm{F})$. Real-world progression-free rate for $\mathrm{P}+\mathrm{Al}$ was $69.8 \%(46.8 \%)$ at $12(24)$ months (P+F: $43.5 \%$ [39.9\%]) months. Real-world survival rate was $89.8 \%$ (71.4\%) at 12 (24) months (P+F: 76.3\% [65.0\%]). Conclusion: The study findings are consistent with previous studies of palbociclib-based therapy.

Lay abstract: Aim: Palbociclib, a drug that inhibits cancer cell growth, is approved to treat hormone receptor-positive, HER- advanced breast cancer in combination with hormone therapy drugs, such as aromatase inhibitor $(\mathrm{P}+\mathrm{Al})$ or fulvestrant $(\mathrm{P}+\mathrm{F})$. Clinical trials indicate that palbociclib is effective in patients with HR+/HER- advanced breast cancer; however, evidence from real-life use of these drugs is limited. Materials \& methods: This study reviewed the medical records of patients who received $\mathrm{P}+\mathrm{Al}$ or $\mathrm{P}+\mathrm{F}$ in US community oncology clinics. Results: After 12 months of treatment with P+Al, 90 in 100 women were still alive and 70 in 100 were living without their cancer getting worse; of those patients who received $\mathrm{P}+\mathrm{F}, 76$ in 100 were still alive and 44 in 100 were living without their cancer getting worse. Conclusion: The findings from this study are consistent with previous studies of palbociclib.

First draft submitted: 23 July 2020; Accepted for publication: 20 October 2020; Published online: 10 November 2020

Keywords: advanced breast cancer • metastatic breast cancer • palbociclib • real-world data • real-world progression-free rate $\bullet$ real-world progression-free survival • real-world survival rate $\bullet$ real-world tumor response

Hormone receptor-positive $(\mathrm{HR}+)$ and HER2- breast cancer are the most common breast cancer subtype in the USA, accounting for $73 \%$ of all breast cancers [1,2]. Although women with early-stage breast cancer generally have a good prognosis, women with distant metastases have a 5-year relative survival rate of $27 \%$ [2].

Endocrine therapy, including aromatase inhibitors (AIs), have been a mainstay for patients with HR+/HER2locally advanced or metastatic breast cancer $(\mathrm{aBC} / \mathrm{mBC})$ [3-5]. The long-term success of these therapies is limited by high rates of primary or acquired resistance to hormonal therapies [6]. Evolving understanding of breast cancer biology and resistance mechanisms to endocrine treatment has led to the development of CDK4/6 inhibitors which interrupt the cell cycle and in combination with endocrine partners have expanded treatment options for women with HR+/HER2- aBC/mBC.

The first CDK4/6 inhibitor approved was palbociclib (P) (Ibrance ${ }^{\circledR}$; Pfizer Inc., NY, USA), which gained accelerated US FDA approval in February 2015 based on the Phase I/II PALOMA-1 trial and full approval with 
the confirmatory Phase III trial PALOMA-2. In PALOMA-2, women with estrogen receptor-positive/HER2- aBC were randomized to receive palbociclib in combination with letrozole versus letrozole in combination with placebo as first-line therapy. The palbociclib combination arm had a median progression-free survival (PFS) of 24.8 months (95\% CI: 22.1-not estimable) versus 14.5 months (95\% CI: 12.9-17.1) for the letrozole + placebo arm (hazard ratio for disease progression or death $=0.58 ; 95 \%$ CI: 0.46-0.72) [7]. In the Phase III trial PALOMA-3, women with $\mathrm{HR}+/ \mathrm{HER} 2-\mathrm{aBC} / \mathrm{mBC}$ who progressed after prior endocrine treatment randomized to receive a combination of palbociclib with fulvestrant $(\mathrm{P}+\mathrm{F})$ had a median PFS (9.5 months; 95\% CI: 9.2-11.0) that was statistically superior to women who received fulvestrant + placebo (4.6 months; $95 \%$ CI: 3.5-5.6) (hazard ratio $=0.46 ; 95 \%$ CI: 0.36-0.59) [8]. Palbociclib and two additional CDK4/6 inhibitors approved subsequently, ribociclib (Kisqali ${ }^{\circledR}$, Novartis, NJ, USA) and abemaciclib (Verzenio ${ }^{\circledR}$, Lilly, IN, USA), have become a standard of care based on data from seven randomized controlled trials demonstrating PFS benefit and more recently, evidence of overall survival benefit.

The present study contributes to a growing body of real-world evidence of treatment patterns and clinical effectiveness of palbociclib in real-world settings [9-18]. The objective of this study was to describe patient demographics, clinical characteristics, real-world treatment patterns and effectiveness among women receiving $\mathrm{P}+\mathrm{AI}$ as initial endocrine-based therapy or $\mathrm{P}+\mathrm{F}$ after failure of prior endocrine therapy for the treatment of HR+/HER2$\mathrm{aBC} / \mathrm{mBC}$. Patients receiving $\mathrm{P}+\mathrm{F}$ could have failed endocrine therapy in the adjuvant setting and received $\mathrm{P}+\mathrm{F}$ as first-line $\mathrm{mBC}$ or have failed endocrine therapy in the metastatic setting and received $\mathrm{P}+\mathrm{F}$ as second-line $\mathrm{mBC}$. Patients initiating $\mathrm{P}+\mathrm{F}$ in third or later lines were excluded from data abstraction. Clinical outcomes that were evaluated include real-world PFS (rwPFS), real-world progression-free rate (rwPFR), real-world survival rate (rwSR) and real-world tumor response. Subgroup analyses by metastasis site were also performed.

\section{Materials \& methods}

Study design

This was a retrospective, database analysis of women with $\mathrm{HR}+/ \mathrm{HER} 2-\mathrm{aBC} / \mathrm{mBC}$ who received a regimen of $\mathrm{P}+\mathrm{AI}$ or P+F between 3 February 2015 (month that palbociclib received FDA approval) and 31 July 2017. Data cut off was 31 December 2017. Eligible patients were identified and accrued from the Vector Oncology Data Warehouse (VODW), a repository of aggregated demographic, medical, treatment and patient-reported outcomes data from a network of ten community oncology practices in the USA. The protocol for this study received institutional review board approval from IntegReview (TX, USA).

\section{Study population}

Patients for this study were identified through structured query language queries of the VODW. The inclusion criteria required: ICD-9 code of 174.x or ICD-10 code of C50.x (malignant neoplasm of breast); diagnosis confirmed by clinical review; diagnosis of advanced (stage IIIB or IIIC) breast cancer or stage IV breast cancer or identified as having distant metastases at any point in the patient record; female sex; and age $\geq 18$ years old at the time of qualifying diagnosis. Eligible patients had to be confirmed to have: record of estrogen receptor-positive or progesterone receptor-positive disease; no record or indication of HER2+ disease or confirmation of HER2- status; and initiation of on-label P+AI (first-line endocrine therapy) or P+F (after failure of prior endocrine therapy) between 3 February 2015 and 31 July 2017. Data cut off was 31 December 2017. Patients with off-label use of palbociclib (defined as $\mathrm{P}+\mathrm{AI}$ not as the first-line users or taking $\mathrm{P}+\mathrm{F}$ without prior endocrine therapy records) were not included in the analysis. Eligibility was verified by clinical research nurse (CRN) review.

\section{Data source \& extraction}

This study's data source was secondary data from the VODW, drawn from existing clinical data originally collected as part of routine care. The database is a repository that includes electronic medical record data from ten US community oncology practices, representing more than 30 practice locations. These represent physician-led practices that draw patients from all 48 contiguous states, with a concentration of patients from the southern states. In addition to geographic variability, these practices also vary in community settings, with both rural and urban practices being included, and range in size from as small as two to as large as 20 physicians. The VODW includes provider notes to support the collection of key information not otherwise available in structured electronic medical record fields, such as verification of disease progression. VODW is a comprehensive cancer patient database comprising demographic, clinical, treatment and patient-reported outcome variables from more than 180,000 cancer patients. In addition, 
CRNs abstracted key data related to patient characteristics, clinical characteristics and certain treatment outcomes (e.g., occurrence of disease progression, metastatic diagnosis and tumor response), from provider notes that were not otherwise available in structured data fields and linked these unstructured data to standard field data. All data abstracted from the VODW by CRNs underwent an independent quality assurance review. Data housed in electronic case report forms were exported to SAS datasets for data cleaning and preparation, creation of derived values and preparation of analysis datasets. Data then underwent initial statistical review including examination of all fields for outlier values and evaluation for internal consistency of study data. Values that were flagged as potentially anomalous during statistical review were queried and resolved before analysis datasets were finalized. All study data also underwent scientific review of study results prior to data lock.

The main objectives of this study were to describe patient demographics, clinical characteristics and real-world treatment patterns among female patients receiving $\mathrm{P}+\mathrm{AI}$ or $\mathrm{P}+\mathrm{F}$ as treatment of $\mathrm{HR}+/ \mathrm{HER} 2-\mathrm{aBC} / \mathrm{mBC}$. Primary outcomes assessed in this study were rwPFS, rwPFR and rwSR. rwPFS was defined as the interval from the start of a line of therapy until the occurrence of disease progression, death or end of the medical record, whichever occurred first. Dates of all disease progressions after diagnosis of $\mathrm{aBC} / \mathrm{mBC}$ were directly determined from radiological scan notes and/or medical progress notes. If the radiological scan notes and provider documentation were not consistent, progression diagnosis was based on provider documentation. Dates of death were determined from the medical record and Social Security National Death Index. Additionally, rwPFR and rwSR were assessed at $6,12,18$ and 24 months. If a patient had not died at the time of data cut off (31 December 2017), the patient was censored.

Tumor response classifications were assessed as best overall response within regimen for the respective line of therapy. Response classifications were made based on the treating physician's statement of response and/or radiological scan notes. If the radiological scan notes and provider documentation were not consistent, response classification was based on provider documentation. Real-world classification included the following criteria: real-world complete response (rwCR), real-world partial response (rwPR), real-world stable disease, real-world progressive disease and not evaluable, based on the treating physician's statements. If real-world tumor response information was missing, the status was classified as 'undocumented'. A status of either not evaluable or undocumented was considered immeasurable and was not included in the real-world response rate calculation.

\section{Analysis}

Descriptive analyses were performed; no formal hypothesis was tested. Descriptive analysis was conducted for patient demographics and clinical characteristics, treatment and dosing patterns, clinical outcomes and real-world tumor response assessed in this study. Unadjusted rwPFS was calculated using Kaplan-Meier methods. The KaplanMeier product limit estimator was employed to descriptively characterize time-to-event outcomes. Real-world overall survival was not calculated due to limited follow-up time. rwPFR and rwSR were assessed at $6,12,18$ and 24 months. Best tumor response was assessed in each progression-based line of therapy. Results were grouped by treatment regimen: $\mathrm{P}+\mathrm{AI}$ or $\mathrm{P}+\mathrm{F}$. A supplemental analysis based on the following metastatic site grouping was performed: visceral (metastasis at one or more of the following sites: liver, pancreas, intestines, lung, heart, malignant pleural effusion, peritoneum, pleura or other visceral); nonvisceral (metastasis at one or more of the following sites: bone, brain, skin, chest wall, contralateral breast or distant lymph nodes); or bone only (bone metastasis, with no metastasis at any other identified site).

\section{Results}

\section{Patients}

Medical records for 304 patients diagnosed with $\mathrm{aBC} / \mathrm{mBC}$ after prior endocrine therapy for breast cancer were initially abstracted. The final analysis included 281 (92.4\%) patients who received palbociclib per labeled indication (Table 1). Most of these 281 patients were treated with a combination of $\mathrm{P}+\mathrm{AI}$ as first-line treatment in the metastatic setting $(\mathrm{n}=233 ; 82.9 \%)$, and the remaining patients were treated with a combination of $\mathrm{P}+\mathrm{F}$ after having previously failed endocrine therapy $(n=48 ; 17.1 \%)$. Five of the 233 patients who received P+AI did not have a record for the sites of metastases; therefore, results by sites of metastases are reported for 276 patients.

The majority of patients included in the analysis had a mean (standard deviation) age of 63.4 (11.2) and median age of 64.5. Only $22(7.8 \%)$ patients had an Eastern Cooperative Oncology Group Performance Status $\geq 2$. The most common sites of distant metastases present at initiation of $\mathrm{P}$-containing regimens were bone $(\mathrm{n}=219 ; 77.9 \%)$, followed by distant lymph node(s) (26.3\%), lung (23.8\%) and liver (18.1\%). 
Table 1. Demographic and clinical characteristics at baseline ${ }^{\dagger}$

\begin{tabular}{|c|c|c|c|}
\hline Variables & $\begin{array}{l}\text { Palbociclib + aromatase inhibitor } \\
(n=233)\end{array}$ & $\begin{array}{l}\text { Palbociclib + fulvestrant } \\
(n=48)\end{array}$ & $\begin{array}{l}\text { Overall } \\
(n=281)\end{array}$ \\
\hline \multicolumn{4}{|l|}{ Age (years) } \\
\hline$-n$ & 233 & 48 & 281 \\
\hline - Mean (SD) & $62.8(11.35)$ & $66.3(9.89)$ & $63.4(11.18)$ \\
\hline - Median & 63.7 & 66.8 & 64.5 \\
\hline - Min, max & $30.6,86.0$ & $45.6,84.3$ & $30.6,86.0$ \\
\hline \multicolumn{4}{|l|}{ Race, n (\%) } \\
\hline - Black or African-American & $49(21.0 \%)$ & $8(16.7 \%)$ & $57(20.3 \%)$ \\
\hline - White & $173(74.2 \%)$ & $37(77.1 \%)$ & $210(74.7 \%)$ \\
\hline - Other & $7(3.0 \%)$ & $3(6.3 \%)$ & $10(3.6 \%)$ \\
\hline - Unknown/undocumented & $4(1.7 \%)$ & 0 & $4(1.4 \%)$ \\
\hline \multicolumn{4}{|l|}{ Insurance category, n (\%) } \\
\hline - Subtotal & $233(100 \%)$ & $48(100 \%)$ & $281(100 \%)$ \\
\hline - Private insurance only & $72(30.9 \%)$ & $7(14.6 \%)$ & $79(28.1 \%)$ \\
\hline - Public insurance only & $53(22.7 \%)$ & $18(37.5 \%)$ & $71(25.3 \%)$ \\
\hline - Public and private insurance & $87(37.3 \%)$ & $21(43.8 \%)$ & $108(38.4 \%)$ \\
\hline - Neither & $6(2.6 \%)$ & 0 & $6(2.1 \%)$ \\
\hline - Unknown/undocumented & $15(6.4 \%)$ & $2(4.2 \%)$ & $17(6.0 \%)$ \\
\hline \multicolumn{4}{|c|}{ Stage of disease at initial diagnosis of $\mathrm{aBC} / \mathrm{mBC}(\%), \mathrm{n}(\%)$} \\
\hline - Stage III & $11(4.7 \%)$ & $5(10.4 \%)$ & $16(5.7 \%)$ \\
\hline - Stage IV & $222(95.3 \%)$ & $43(89.6 \%)$ & $265(94.3 \%)$ \\
\hline \multicolumn{4}{|c|}{ ECOG Performance Status at diagnosis of aBC/mBC, $\mathrm{n}(\%)$} \\
\hline-0 & $82(35.2 \%)$ & $11(22.9 \%)$ & $93(33.1 \%)$ \\
\hline-1 & $60(25.8 \%)$ & $12(25.0 \%)$ & $72(25.6 \%)$ \\
\hline$-2-3$ & $19(8.2 \%)$ & $3(6.3 \%)$ & $22(7.8 \%)$ \\
\hline - Undocumented & $72(30.9 \%)$ & $22(45.8 \%)$ & $94(33.5 \%)$ \\
\hline \multicolumn{4}{|l|}{ Menopausal status, n (\%) } \\
\hline - Premenopause & $23(9.9 \%)$ & $1(2.1 \%)$ & $24(8.5 \%)$ \\
\hline - Perimenopause & $2(<1 \%)$ & $2(4.2 \%)$ & $4(1.4 \%)$ \\
\hline - Postmenopause & $186(79.8 \%)$ & $40(83.3 \%)$ & $226(80.4 \%)$ \\
\hline - Undocumented & $22(9.4 \%)$ & $5(10.4 \%)$ & $27(9.6 \%)$ \\
\hline \multicolumn{4}{|c|}{ Weighted comorbidity index score at diagnosis of $\mathrm{aBC} / \mathrm{mBC}$} \\
\hline$-n$ & 233 & 48 & 281 \\
\hline - Mean (SD) & $0.6(1.17)$ & $0.4(0.68)$ & $0.5(1.10)$ \\
\hline - Median & 0.0 & 0.0 & 0.0 \\
\hline- Min, max & $0.0,7.0$ & $0.0,2.0$ & $0.0,7.0$ \\
\hline \multicolumn{4}{|c|}{ Treatments received between $\mathrm{aBC} / \mathrm{mBC}$ diagnosis and palbociclib initiation, $\mathrm{n}(\%)$} \\
\hline - Surgery & $33(14.2 \%)$ & $13(27.1 \%)$ & $46(16.4 \%)$ \\
\hline - Chemo/targeted therapy & $39(16.7 \%)$ & $16(33.2 \%)$ & $55(19.6 \%)$ \\
\hline - Radiation therapy & $43(18.5 \%)$ & $20(41.7 \%)$ & $63(22.4 \%)$ \\
\hline - Hormone therapy & $2(<1 \%)^{\ddagger}$ & $44(91.7 \%)$ & $46(16.4 \%)$ \\
\hline - No prior treatment & $145(62.2 \%)$ & $3(6.3 \%)$ & $148(52.7 \%)$ \\
\hline \multicolumn{4}{|c|}{ Sites of distant metastasis at diagnosis of $\mathrm{aBC} / \mathrm{mBC}, \mathrm{n}(\%)$} \\
\hline - Bone only & $88(37.8 \%)$ & $16(33.3 \%)$ & $104(37.0 \%)$ \\
\hline - Visceral & $117(50.2 \%)$ & $28(58.3 \%)$ & $145(51.6 \%)$ \\
\hline - Nonvisceral & $111(47.6 \%)$ & $20(41.7 \%)$ & $131(46.6 \%)$ \\
\hline - Undocumented & $5(2.1 \%)$ & 0 & $5(1.8 \%)$ \\
\hline \multicolumn{4}{|c|}{$\begin{array}{l}\text { †Study baseline was defined as the period within } 3 \text { months before the date of } \mathrm{aBC} / \mathrm{mBC} \text { diagnosis. If information was missing within the defined baseline period, information } \\
\text { for the variable from the date that was closest to the date of } \mathrm{BC} / \mathrm{mBC} \text { diagnosis was used. } \\
\text { ¥Both patients received ovarian suppression treatment, classified as hormone therapy. } \\
\mathrm{aBC} / \mathrm{mBC} \text { : Advanced or metastatic breast cancer; ECOG: Eastern Cooperative Oncology Group; Max: Maximum; Min: Minimum; SD: Standard deviation. }\end{array}$} \\
\hline
\end{tabular}


Table 1. Demographic and clinical characteristics at baseline ${ }^{\dagger}$ (cont.).

\begin{tabular}{|c|c|c|c|}
\hline Variables & $\begin{array}{l}\text { Palbociclib + aromatase inhibitor } \\
(n=233)\end{array}$ & $\begin{array}{l}\text { Palbociclib + fulvestrant } \\
(n=48)\end{array}$ & $\begin{array}{l}\text { Overall } \\
(n=281)\end{array}$ \\
\hline \multicolumn{4}{|c|}{ Sites of distant metastasis at initiation of qualifying palbociclib-containing regimens, $\mathrm{n}(\%)$} \\
\hline$-n$ & 233 & 48 & 281 \\
\hline - Bone & $181(77.7 \%)$ & $38(79.2 \%)$ & $219(77.9 \%)$ \\
\hline - Brain & $5(2.1 \%)$ & 0 & $5(1.8 \%)$ \\
\hline - Chest wall & $15(6.4 \%)$ & $3(6.3 \%)$ & $18(6.4 \%)$ \\
\hline - Contralateral breast & $2(<1 \%)$ & $2(4.2 \%)$ & $4(1.4 \%)$ \\
\hline - Liver & $40(17.2 \%)$ & $11(22.9 \%)$ & $51(18.1 \%)$ \\
\hline - Lung & $57(24.5 \%)$ & $10(20.8 \%)$ & $67(23.8 \%)$ \\
\hline - Malignant pleural effusion & $5(2.1 \%)$ & 0 & $5(1.8 \%)$ \\
\hline - Muscle and soft tissue & $2(<1 \%)$ & $3(6.3 \%)$ & $5(1.8 \%)$ \\
\hline - Peritoneum & $3(1.3 \%)$ & $2(4.2 \%)$ & $6(2.1 \%)$ \\
\hline - Skin & $13(5.6 \%)$ & $3(6.3 \%)$ & $16(5.7 \%)$ \\
\hline - Other visceral & $11(4.7 \%)$ & $2(4.2 \%)$ & $13(4.6 \%)$ \\
\hline - Distant lymph node(s) & $64(27.5 \%)$ & $10(20.8 \%)$ & $74(26.3 \%)$ \\
\hline - Undocumented & $5(2.1 \%)$ & 0 & $5(1.8 \%)$ \\
\hline \multicolumn{4}{|c|}{ Stage of disease at initiation of qualifying palbociclib-containing regimens } \\
\hline - Subtotal & $233(100 \%)$ & $48(100 \%)$ & $281(100 \%)$ \\
\hline - Stage IIIB & $3(1.3 \%)$ & 0 & $3(1.1 \%)$ \\
\hline - Stage IIIC & $2(<1.0 \%)$ & 0 & $2(<1.0 \%)$ \\
\hline - Stage IV & $228(97.9 \%)$ & $48(100 \%)$ & $276(98.2 \%)$ \\
\hline \multicolumn{4}{|c|}{$\begin{array}{l}\text { † Study baseline was defined as the period within } 3 \text { months before the date of aBC/mBC diagnosis. If information was missing within the defined baseline period, information } \\
\text { for the variable from the date that was closest to the date of } \mathrm{aBC} / \mathrm{mBC} \text { diagnosis was used. } \\
\text { ¥Both patients received ovarian suppression treatment, classified as hormone therapy. } \\
\mathrm{aBC} / \mathrm{mBC} \text { : Advanced or metastatic breast cancer; ECOG: Eastern Cooperative Oncology Group; Max: Maximum; Min: Minimum; SD: Standard deviation. }\end{array}$} \\
\hline
\end{tabular}

\section{Treatment/dosing patterns}

$P+A I$

$125 \mathrm{mg} /$ day of palbociclib was the most frequently prescribed starting dose $(\mathrm{n}=199 ; 85.4 \%)$ (Table 2). A total of $69(34.7 \%)$ patients who initiated therapy at $125 \mathrm{mg} /$ day required a dose adjustment; among patients with dose adjustment, $44(63.8 \%)$ reduced to $100 \mathrm{mg} /$ day and $25(36.2 \%)$ reduced to $75 \mathrm{mg} /$ day, respectively.

$P+F$

$125 \mathrm{mg} /$ day of palbociclib was the most frequently prescribed starting dose ( $\mathrm{n}=38 ; 79.2 \%)$. A total of 15 (39.5\%) patients who initiated therapy at $125 \mathrm{mg}$ /day required a dose adjustment; among patients with dose adjustment, eight $(53.3 \%)$ reduced to $100 \mathrm{mg} /$ day and seven $(46.7 \%)$ reduced to $75 \mathrm{mg} /$ day, respectively.

\section{Clinical outcomes}

$P+A I$

The median follow-up for this cohort was 10.8 months. Median rwPFS was 21.2 months (95\% CI: 17.9-not achieved) (Table 3). The rwPFR was $84.8 \%$ at 6 months, $69.8 \%$ at 12 months and $46.8 \%$ at 24 months. The rwSR at 12 months was $89.8 \%$ and $71.4 \%$ at 24 months. Table 4 shows rwPFR and rwSR in patients treated with P+AI at 6,12, 18 and 24 months by metastatic site. Among subgroups based on metastatic sites, the rwPFR was highest for the nonvisceral disease patients at 24 months after palbociclib initiation $(58.8 \%)$. The rwSR was highest for the nonvisceral disease patients at 24 months after palbociclib initiation (76.9\%). For patients who received $\mathrm{P}+\mathrm{AI}$ as the initial endocrine therapy after aBC/mBC diagnosis, 16 (6.9\%) achieved rwCR and 69 (29.6\%) patients achieved rwPR (Table 5). 
Table 2. Dosing information at initiation of palbociclib-containing therapies, by treatment regimen.

\begin{tabular}{|c|c|c|c|}
\hline Treatment pattern & $\begin{array}{l}\text { Palbociclib + aromatase inhibitor } \\
(n=233)\end{array}$ & $\begin{array}{l}\text { Palbociclib + fulvestrant } \\
(n=48)\end{array}$ & $\begin{array}{l}\text { Overall } \\
(n=281)\end{array}$ \\
\hline - Subtotal & $233(100 \%)$ & $48(100 \%)$ & $281(100 \%)$ \\
\hline-100 & $11(4.7 \%)$ & $4(8.3 \%)$ & $15(5.3 \%)$ \\
\hline-75 & $7(3.0 \%)$ & $2(4.2 \%)$ & $9(3.2 \%)$ \\
\hline - Subtotal & $71(100 \%)$ & $17(100 \%)$ & $88(100 \%)$ \\
\hline - From 125 to 100 & $44(62.0 \%)$ & $8(47.1 \%)$ & $52(59.1 \%)$ \\
\hline - From 125 to 75 & $25(35.2 \%)$ & $7(41.2 \%)$ & $32(36.4 \%)$ \\
\hline - From 100 to 75 & $2(2.8 \%)$ & $2(11.8 \%)$ & $4(4.5 \%)$ \\
\hline \multicolumn{4}{|l|}{ Discontinuation, n (\%) } \\
\hline \multicolumn{4}{|c|}{ Reason for dose adjustment ${ }^{\dagger}, \mathrm{n}(\%)$} \\
\hline - Subtotal & $135(100 \%)$ & $32(100 \%)$ & $167(100 \%)$ \\
\hline - Disease progression & $5(2.1 \%)$ & 0 & $5(1.8 \%)$ \\
\hline - Side effects/toxicity & $79(33.9 \%)$ & $18(37.5 \%)$ & $97(34.5 \%)$ \\
\hline - Treatment cost & $1(<1 \%)$ & 0 & $1(<1 \%)$ \\
\hline - Patient refusal/request & $2(<1 \%)$ & $1(2.1 \%)$ & $3(1.1 \%)$ \\
\hline - Other & $45(19.3 \%)$ & $12(25.0 \%)$ & $57(20.3 \%)$ \\
\hline - Undocumented & $3(1.3 \%)$ & $1(2.1 \%)$ & $4(1.4 \%)$ \\
\hline
\end{tabular}

\section{$P+F$}

The median follow-up for this cohort was 7.6 months. Median rwPFS was 11.5 months (95\% CI: 7.0-not achieved) (Table 3). The rwPFR was $67.4 \%$ at 6 months, $43.5 \%$ at 12 months and $39.9 \%$ at 24 months. The rwSR at 12 months was $76.3 \%$ and $65.0 \%$ at 24 months. By metastatic site, the highest rwPFR in patients treated with $\mathrm{P}+\mathrm{F}$ was observed for the bone-only disease patients at both 12 months and 24 months after palbociclib initiation (62.5 and 53.6\%, respectively) (Table 4). The rwSR was highest for the nonvisceral disease patients at 24 months after palbociclib initiation (80.9\%). For patients who received $\mathrm{P}+\mathrm{F}$ after breast cancer diagnosis, two (4.4\%) patients had an rwCR, three (6.3\%) patients had an rwPR and 14 (31.3\%) patients had real-world stable disease (sum of line 1 and 2 for $\mathrm{P}+\mathrm{F}$ ) (Table 5).

\section{Discussion}

This study demonstrates real-world benefit for patients treated with $\mathrm{P}+\mathrm{AI}$ in the first line of therapy and for patients treated with $\mathrm{P}+\mathrm{F}$ after prior endocrine therapy. Limited sample size and follow-up time limit the generalizability and maturity of the data. Limitations that apply to real-world data sources include missing data, inaccurate data capture and variability in documentation between participating practices and physicians. Additional limitations include lack of uniform evaluation schedule and recognized objective criteria to determine tumor response, for example, Response Evaluation Criteria in Solid Tumors guidelines. This study reflects treatment practice patterns within community oncology practices in the USA that are part of the Vector Oncology Network; it is possible that treatment patterns may differ in patients who received care in other settings. Limited time since approval of the $\mathrm{P}+\mathrm{F}$ cohort limited the duration of follow-up for this cohort. Despite these limitations, the current study provides further insight into the real-world use of palbociclib in US patients with aBC/mBC. 


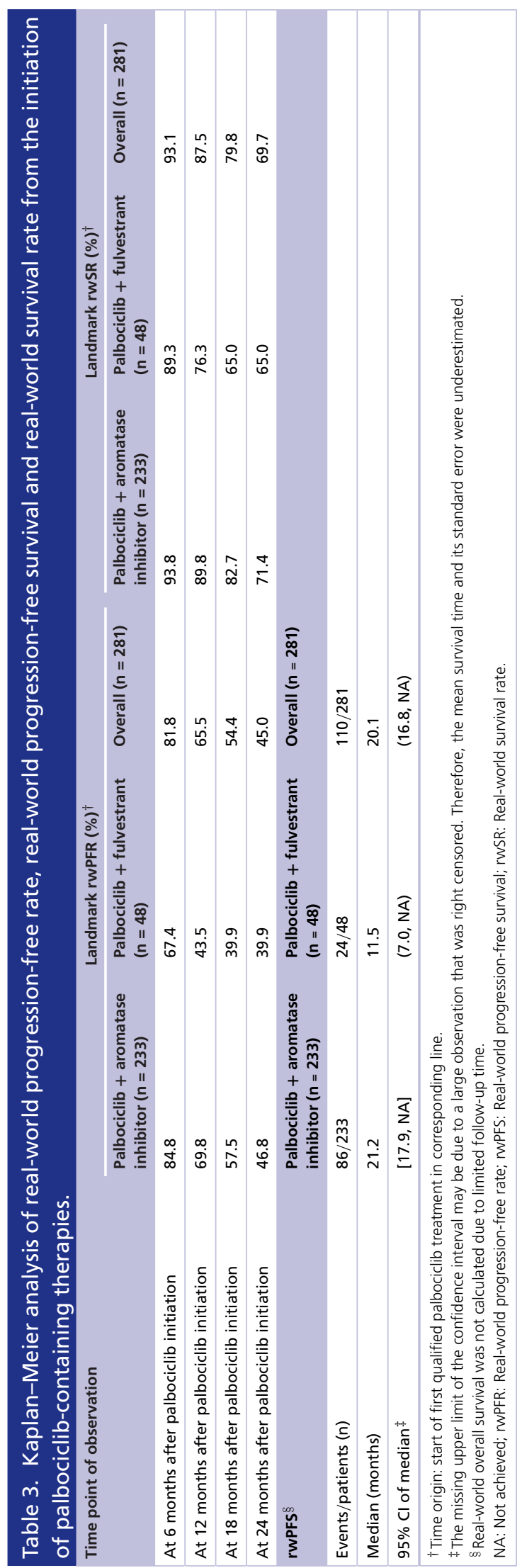




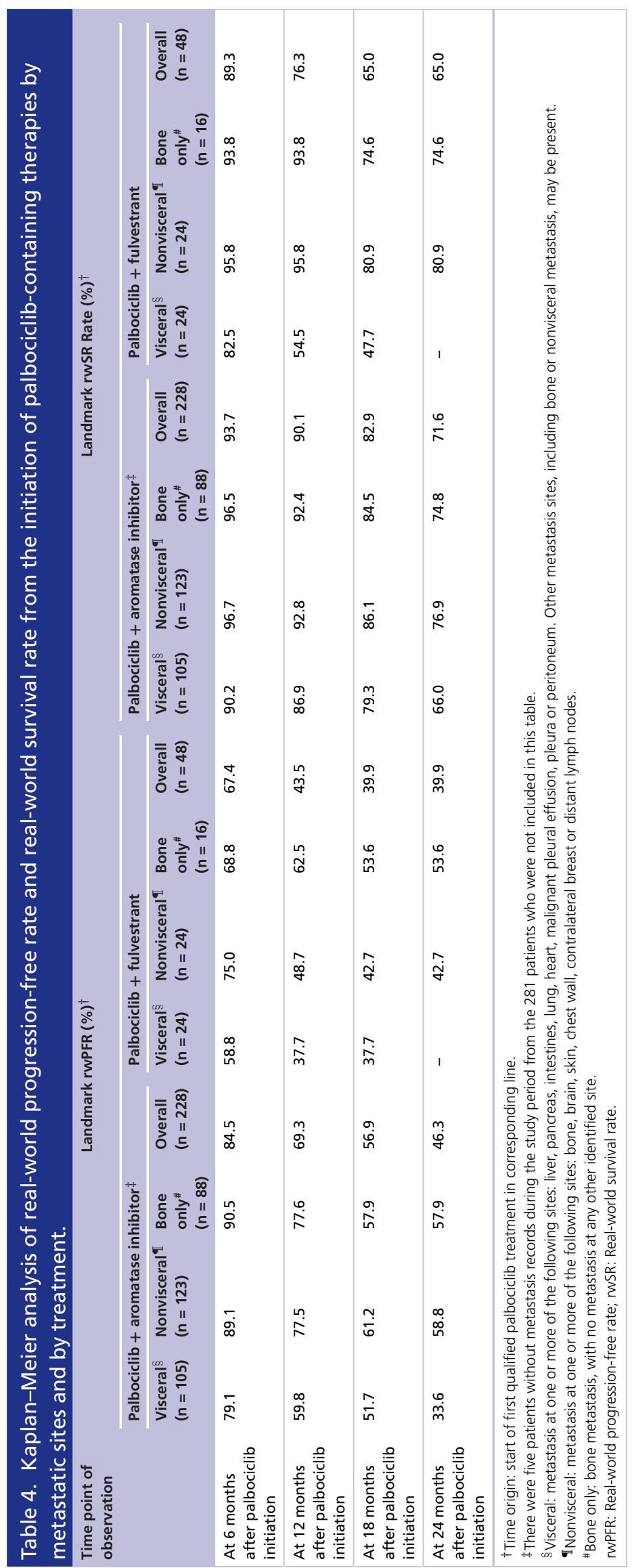


Table 5. Best response ${ }^{\dagger}$ in patients receiving palbociclib treatment for advanced/metastatic breast cancer at each line of therapy.

\begin{tabular}{|c|c|c|c|}
\hline \multirow[t]{2}{*}{ Variable/statistic } & \multirow{2}{*}{$\begin{array}{l}\text { Palbociclib + aromatase inhibitor } \\
\qquad(n=233)\end{array}$} & \multicolumn{2}{|c|}{ Palbociclib + fulvestrant $(n=48)$} \\
\hline & & Line $1(n=3)$ & Line $2(n=45)$ \\
\hline Real-world complete response, $\mathrm{n}(\%)$ & $16(6.9 \%)$ & 0 & $2(4.4 \%)$ \\
\hline Real-world partial response, $\mathrm{n}(\%)$ & $69(29.6 \%)$ & $1(33.3 \%)$ & $2(4.4 \%)$ \\
\hline Real-world stable disease, n (\%) & $27(11.6 \%)$ & $1(33.3 \%)$ & $13(28.9 \%)$ \\
\hline Real-world progressive disease, $\mathrm{n}(\%)$ & $27(11.6 \%)$ & 0 & $9(20.0 \%)$ \\
\hline Other $\ddagger, n(\%)$ & $71(30.5 \%)$ & $1(33.3 \%)$ & $13(28.9 \%)$ \\
\hline Undocumented, n (\%) & $23(9.9 \%)$ & 0 & $6(13.3 \%)$ \\
\hline Subtotal & 233 & 3 & 45 \\
\hline
\end{tabular}

\section{Conclusion}

This study provides a real-world assessment of treatment patterns and clinical effectiveness outcomes of patients with $\mathrm{HR}+/ \mathrm{HER} 2-\mathrm{aBC} / \mathrm{mBC}$ who received $\mathrm{P}+\mathrm{AI}$ or $\mathrm{P}+\mathrm{F}$ in a network of community oncology practices. Findings supplement those previously reported in clinical trials and are consistent with other real-world studies demonstrating the benefit of palbociclib combination therapy in a community oncology setting.

\section{Summary points}

- This retrospective observational study examined 281 patients with hormone receptor-positive/HER- advanced or metastatic breast cancer who received on-label palbociclib-based treatment from 3 February 2015 to 31 December 2017, to provide a description of patient characteristics, treatment patterns and clinical outcomes in real-world community oncology treatment settings.

- More than $80 \%$ of the patients were treated with palbociclib + aromatase inhibitor $(\mathrm{P}+\mathrm{Al})$ as first-line treatment in the advanced/metastatic setting. Only a small proportion of patients were treated with palbociclib + fulvestrant $(\mathrm{P}+\mathrm{F})$ after having previously failed endocrine therapy.

- The study found that the most common sites of distant metastases present at initiation of palbociclib-based therapy were bone, followed by distant lymph node(s), lung and liver.

- The study showed the most frequently prescribed palbociclib starting dose for P+Al was $125 \mathrm{mg} /$ day. Among these patients, more than $30 \%$ required a palbociclib dose reduction to either 100 or $75 \mathrm{mg} /$ day. The most frequently prescribed palbociclib starting dose for $\mathrm{P}+\mathrm{F}$ was $125 \mathrm{mg} /$ day. Among these patients, more than $35 \%$ required a P-dose reduction to either 100 or $75 \mathrm{mg} /$ day.

- $\mathrm{P}+\mathrm{F}$ was approved 1 year later than $\mathrm{P}+\mathrm{Al}$, so there was limited opportunity to observe other outcomes from this cohort. The study results in this cohort may not generalize to a more recent cohort or to patients treated outside the community oncology setting.

- The study estimated median real-world progression-free survival, real-world progression-free rate (rwPFR) and real-world survival rate align with those previously reported in clinical trials and other observational studies. These findings demonstrate the benefit of palbociclib combination therapy in the community oncology setting.

- In the P+Al cohort, the rwPFR was highest for the nonvisceral disease patients at 24 months after palbociclib initiation. The real-world survival rate was highest for the nonvisceral disease patients at 24 months after palbociclib initiation.

- For patients who received $\mathrm{P}+\mathrm{Al}$ as the initial endocrine therapy after advanced or metastatic breast cancer diagnosis, more than $35 \%$ of patients achieved positive response (real-world complete response and real-world partial response).

- These data provide an important benchmark for understanding the current characteristics of patients who received P-based therapy and their treatment patterns. It also provides new insight for clinical outcomes including rwPFS, rwPFR and tumor response rate for the treated patients.

\section{Author contributions}

D Mitra, J Lin, MD Fisher, N Hu, C Davis and MS Walker contributed to the study design. N Hu participated in the collection and assembly of data. All authors had full access to the data, and all authors contributed to the drafting, critical review and revision of the manuscript. All authors granted approval of the final manuscript for submission. 
Financial \& competing interests disclosure

J Cueto and D Spurden are employees of Pfizer Inc. and may own stock or options in the company contributed to data analysis and editorial support. J Trocio was a previous employee of Pfizer Inc., and contributed to the study design. Data collection was undertaken by Vector Oncology as part of this study. Pfizer Inc., sponsored this study and provided financial support for the conduct of the research and for preparation of the article. L McRoy and D Mitra are employees of Pfizer Inc., and may own stock or options in the company. J Lin, MD Fisher, N Hu, C Davis and MS Walker are employees of ConcertAl and were paid consultants to Pfizer Inc., in connection with the development of this manuscript. The authors have no other relevant affiliations or financial involvement with any organization or entity with a financial interest in or financial conflict with the subject matter or materials discussed in the manuscript apart from those disclosed.

Medical writing and editorial support were provided by A Swartz, an employee of Speaking of Science and was funded by Pfizer Inc.

Ethical conduct of research

The authors state that they have obtained appropriate institutional review board approval from IntegReview (TX, USA).

Data sharing statement

ConcertAl does not make datasets publicly available because study data are used under license from source practices. ConcertAl will consider requests to access study datasets on a case-by-case basis.

Previous publication

A portion of this research was presented at the Florida Society of Clinical Oncology (FLASCO) 2018 Fall Session, 8-10 November 2018 and at the 41st Annual San Antonio Breast Cancer Symposium (SABCS), 4-8 December 2018.

Open access

This work is licensed under the Attribution-NonCommercial-NoDerivatives 4.0 Unported License. To view a copy of this license, visit http://creativecommons.org/licenses/by-nc-nd/4.0/

\section{References}

Papers of special note have been highlighted as: $\bullet$ of interest; $\bullet \bullet$ of considerable interest

1. Howlader N, Altekruse SF, Li CI et al. US incidence of breast cancer subtypes defined by joint hormone receptor and HER2 status. J. Natl Cancer Inst. 106(5), dju055 (2014).

2. American Cancer Society. Breast cancer facts \& figures 2019-2020. (2019). https://www.cancer.org/content/dam/cancer-org/research/c ancer-facts-and-statistics/breast-cancer-facts-and-figures/breast-cancer-facts-and-figures-2019-2020.pdf

3. Cardoso F, Senkus E, Costa A et al. 4th ESO-ESMO International Consensus guidelines for advanced breast cancer (ABC 4). Ann. Oncol. 29(8), 1634-1657 (2018).

- Describes the European School of Oncology (ESO) European Society for Medical Oncology (ESMO) treatment guideline for advanced breast cancer with endocrine therapy.

4. National Comprehensive Cancer Network. NCCN clinical practice guidelines in oncology (NCCN guidelines ${ }^{\circledR}$ ). Breast cancer. Version 4.2020. (2020). https://www.nccn.org/professionals/physician_gls/pdf/breast.pdf

- Describes current National Comprehensive Cancer Network (NCCN) treatment guidelines in this population.

5. Shah AN, Cristofanilli M. The growing role of CDK4/6 inhibitors in treating hormone receptor-positive advanced breast cancer. Curr. Treat. Options Oncol. 18(1), 6 (2017).

6. Verma S, Bartlett $\mathrm{CH}$, Schnell $\mathrm{P}$ et al. Palbociclib in combination with fulvestrant in women with hormone receptor-positive/HER2-negative advanced metastatic breast cancer: detailed safety analysis from a multicenter, randomized, placebo-controlled, Phase III study (PALOMA-3). Oncologist 21(10), 1165-1175 (2016).

7. Finn RS, Martin M, Rugo HS et al. Palbociclib and letrozole in advanced breast cancer. N. Engl. J. Med. 375(20), 1925-1936 (2016).

8. Cristofanilli M, Turner NC, Bondarenko I $e$ al. Fulvestrant plus palbociclib versus fulvestrant plus placebo for treatment of hormone-receptor-positive, HER2-negative metastatic breast cancer that progressed on previous endocrine therapy (PALOMA-3): final analysis of the multicentre, double-blind, Phase III randomised controlled trial. Lancet Oncol. 17(4), 425-439 (2016).

9. Brufsky A, Mitra D, Davis KL et al. Treatment patterns and outcomes associated with palbociclib plus letrozole for postmenopausal women with HR(+)/HER2(-) advanced breast cancer enrolled in an expanded access program. Clin. Breast Cancer 19(5), 317-325 (2019).

10. Eziokwu AS, Varella L, Kruse ML et al. Real-world clinical outcomes of palbociclib in hormone receptor positive (HR+) metastatic breast cancer (MBC) patients. J. Clin. Oncol. 36(Suppl. 15), e13034 (2018). 
11. Kish JK, Ward MA, Garofalo D et al. Real-world evidence analysis of palbociclib prescribing patterns for patients with advanced/metastatic breast cancer treated in community oncology practice in the USA 1 year post approval. Breast Cancer Res. 20(1), 37 (2018).

-• Describes an observational study on real-world palbociclib $(\mathbf{P})$ prescribing pattern using real-world data.

12. Li N, Du EX, Chu L et al. Real-world palbociclib dosing patterns and implications for drug costs in the treatment of HR+/HER2metastatic breast cancer. Expert Opin. Pharmacother. 18(12), 1167-1178 (2017).

-• Describes an observational study on real-world $P$ prescribing pattern using real-world data.

13. Malik F, Ali N, Jafri SIM, Sundermeyer ML, Seidman MJ, Fidler CJ. Real-world experience of palbociclib in hormone receptor-positive metastatic breast cancer. J. Clin. Oncol. 35(Suppl. 15), e18094 (2017).

14. Taylor-Stokes G, Mitra D, Waller J, Gibson K, Milligan G, Iyer S. Treatment patterns and clinical outcomes among patients receiving palbociclib in combination with an aromatase inhibitor or fulvestrant for HR+/HER2-negative advanced/metastatic breast cancer in real-world settings in the US: results from the IRIS study. Breast 43, 22-27 (2019).

-• Describes an observational study on treatment patterns and clinical outcomes for patients who received $P$ containing therapy using real-world data.

15. Waller J, Mitra D, Mycock K et al. Real-world treatment patterns and clinical outcomes in patients receiving palbociclib for hormone receptor-positive, human epidermal growth factor receptor 2-negative advanced or metastatic breast cancer in Argentina: the IRIS study. J. Glob. Oncol. 5, JGO1800239 (2019).

-• Describes an observational study on treatment patterns and clinical outcomes for patients who received $\mathbf{P}$ containing therapy using real-world data.

16. Watson GA, Deac O, Aslam R, O’Dwyer R, Sukor S, Kennedy MJ. A real-world experience of palbociclib-induced adverse events in women with HR-positive/Her2-negative metastatic breast cancer. J. Clin. Oncol. 36(Suppl. 15), e13061 (2018).

17. Zurcher J-P, Aedo V, Stravodimou A, Sarivalasis A, Zaman K. Efficacy and safety of the CDK4/6 inhibitor palbociclib during different lines of treatment in patients with advanced stage endocrine sensitive/HER2 negative breast cancer. J. Clin. Oncol. 36(Suppl. 15), e13052 (2018).

18. McRoy L, Mardekian J, Trocio J. Real-world clinical outcomes in patients treated with first-line palbociclib in combination endocrine partner at 2 years postapproval. Presented at: 35th Miami Breast Cancer Conference. FL, USA (8-11 March 2018). 